\title{
Using Spontaneous Eye-blink Rates to Predict the Motor Status of Patients with Parkinson's Disease
}

\author{
Hirotaka Iwaki ${ }^{1}$, Hiroyuki Sogo ${ }^{2}$, Haruhiko Morita ${ }^{3}$, Noriko Nishikawa ${ }^{1}$, Rina Ando ${ }^{1}$, \\ Noriyuki Miyaue ${ }^{1}$, Satoshi Tada ${ }^{1}$, Hayato Yabe ${ }^{1}$, Masahiro Nagai ${ }^{1}$ and Masahiro Nomoto ${ }^{1}$
}

\begin{abstract}
:
Objective Assessing daily motor fluctuations is an important part of the disease management for patients with Parkinson's disease (PD). However, the frequent recording of subjective and/or objective assessments is not always feasible, and easier monitoring methods have been sought. Previous studies have reported that the spontaneous eye-blink rate (EBR) is correlated with the dopamine levels in the brain. Thus, the continuous monitoring of the EBR may be useful for predicting the motor status in patients with PD.

Methods Electrooculograms (EOGs) were recorded for up to 7.5 hours from three PD patients using a wearable device that resembled ordinary glasses. An receiver operating characteristic (ROC) analysis was performed to compare the ability of the EBR estimates at each time-point (Blink Index) and the plasma levodopa levels to predict the motor status.

Results The Blink Index was correlated with the plasma levodopa levels. When an indicator for the first hour of the observation period was included in the model, the Blink Index discerned wearing-off and dyskinesia as accurately as the plasma levodopa level.

Conclusion Our study provides preliminary evidence regarding the utility of continuous EBR monitoring for the non-invasive evaluation of the motor status in patients with PD.
\end{abstract}

Key words: Parkinson's disease, blink, motor fluctuations, wearable electronic device, wearing-off: dyskinesia

(Intern Med 58: 1417-1421, 2019)

(DOI: 10.2169/internalmedicine.1960-18)

\section{Introduction}

Levodopa is a gold standard for the treatment of Parkinson's disease (PD). However, as the disease progresses, presynaptic handling and storage of levodopa-derived dopamine are reduced, and the strength of dopaminergic stimulation gradually becomes dependent on plasma levodopa levels (1). At this stage, patients typically experience overstimulation (dyskinesia) at peak plasma levodopa levels, followed by insufficient dopaminergic stimulation at trough levels (wearing-off). Although it is important to stabilize the plasma levodopa level, the half-life of levodopa is only 1-2 hours, and its absorption is affected by various factors, including age, stomach contents, gastrointestinal activity, kid- ney function, and concomitant medications (2-4).

Measuring plasma levodopa levels and investigating the levodopa pharmacokinetics in each patient provides substantial information that can be used to personalize the dosage and frequency of medication. However, as the associated procedures require multiple blood samples, their use in the clinical setting is limited, necessitating the development of less invasive clinical monitoring strategies. Previous studies have reported that the spontaneous eye-blink rate (EBR) is correlated with the level of dopamine in the brain $(5,6)$. Slower blinking rates have been reported in patients with $\mathrm{PD}$, and both levodopa treatment and deep brain stimulation of the subthalamic nucleus have been shown to increase EBRs $(6,7)$. Thus, EBRs may represent an inexpensive, non-invasive method for monitoring dopaminergic activity in

${ }^{1}$ Department of Neurology and Clinical Pharmacology, Ehime University Graduate School of Medicine, Japan, ${ }^{2}$ Faculty of Law and Letters, Ehime University, Japan and ${ }^{3}$ EdiGENE Corporation, Japan

Received for publication August 16, 2018; Accepted for publication November 1, 2018

Correspondence to Dr. Hirotaka Iwaki, h-iwaki@m.ehime-u.ac.jp 
Table. The Summary Characteristics of the Patients.

\begin{tabular}{lccc}
\hline & Case 1 & Case 2 & Case 3 \\
\hline Age, years & 45.1 & 66.9 & 72.2 \\
Sex & Male & Female & Female \\
Disease Duration, years & 8.6 & 8.7 & 9.8 \\
Modified Hoehn and Yahr stage (On) & 2.5 & 2.5 & 3 \\
Body Mass Index, kg/m² & 24.4 & 20.6 & 22.2 \\
Levodopa dose, mg/day & 200 & 500 & 450 \\
Levodopa equivalent dose*, mg/day & 265 & 852 & 699 \\
Blink Index, Mean (SD) & $196(158)$ & $434(166)$ & $159(61)$ \\
\hline
\end{tabular}

* Levodopa equivalent dose were calculated based on the reference 8 (Adapted from

Tomlinson CL, et al. Mov Disord 2010;25:2649-2653).

the brain. However, no studies have evaluated the clinical significance of spontaneous EBRs for monitoring motor fluctuations in patients with PD.

The present study aimed to provide preliminary data in this area using a wearable electrooculography (EOG) device resembling a pair of eyeglasses. The EOG device was used to record EBRs in patients with PD in real-life settings. Thereafter, the ability of EBRs to predict the motor status was compared to that of plasma levodopa levels.

\section{Materials and Methods}

Three Japanese patients with wearing-off and dyskinesia participated in the study. All participants provided their written informed consent to participate in the present study. The protocol was approved by the local institutional review board of clinical research ethics.

All patients wore an eyeglasses-shaped device (JINS MEME $^{\circledR}$. JINS, Tokyo, Japan) for up to 7.5 hours from the morning until the evening. The glasses were wirelessly connected to a PC, sending real-time EOG data at a rate of 50 Hz. EOG data were recorded from three EOG sensors: one was placed between the eyebrows and one was placed on either side of the nose. Venous blood samples were also obtained from each patient prior to taking medication, every 15 minutes up to 1 hour from taking the medication, and every 30 to 60 minutes thereafter. The plasma levodopa levels were measured using high-performance liquid chromatography (HPLC), as previously described (4). Motor symptoms (wearing-off and negative/positive dyskinesia) were also assessed by a specialized neurologist and recorded each time blood was drawn. During the study, the patients were free to engage in normal activities, except for sleeping. Patients engaged in activities such as reading, watching TV, walking, eating, and talking.

The EOG data were used to calculate a Blink Index for each 5-min interval during the study. The Blink Index was calculated according to the following algorithm: 1) At each time-point, EOG data were obtained for a 10-minute window, ranging from 5 minutes before the time-point to $5 \mathrm{~min}$ utes after the time-point. 2) Within the 10-minute window, the mean and standard deviation (SD) of the amplitude of the horizontal EOG data were calculated. 3) Whenever the amplitude exceeded the mean by $+1 \mathrm{SD}$, the time until the EOG signal fell below the mean $+1 \mathrm{SD}$ was recorded $(+1$ SD exceeding time). 4) If the $+1 \mathrm{SD}$ exceeding time was between 100 and $300 \mathrm{~ms}$ it was counted as a "blink". 5) Finally, the number of blinks within the window was multiplied by six and converted to the Blink Index, to estimate the blink rate per hour at a given time-point. The algorithm was validated prior to the study using accurate, videorecorded blinking data.

To illustrate the utility of blink monitoring, we constructed a multivariate logistic regression model of the EBRs in order to predict wearing-off. Because the EBRs varied among individuals, we adjusted the EBR for each participant. The receiver operating characteristic (ROC) curve for the model was compared with the equivalent model using the plasma levodopa levels, via the Diebold-Mariano test. We applied a similar analysis to the dyskinesia outcomes. In addition, we conducted a post hoc analysis adding an indicator variable for the first one hour in the models. The aim of the analysis was to assess the potential influence of the first one hour on these predictions considering the frequent blood samplings and patient discomfort prior to becoming used to the device. A statistical analysis was performed and data were plotted using the $\mathrm{R}$ software program (version 3.4.2 for Windows 64 bit). Two-tailed $p$ values of $<0.05$ were considered to indicate statistical significance.

\section{Results}

The characteristics of the three patients are presented in Table (8). Fig. 1 shows the associations among the motor status, Blink Index, and plasma levodopa levels for each patient. The average Blink Index varied across individuals as well as within individuals; however, the overall changes in the Blink Index within individuals were well correlated with the plasma levodopa levels (Fig. 1).

The ROC curves for the prediction of wearing-off demonstrated that the Blink Index (ROC of 0.793) had a significantly lower predictive value relative to the plasma levodopa levels ( $\mathrm{p}<0.001$ ), which exhibited an area under the curve (AUC) value of 0.946 (Fig. 2). However, when adjusted for 


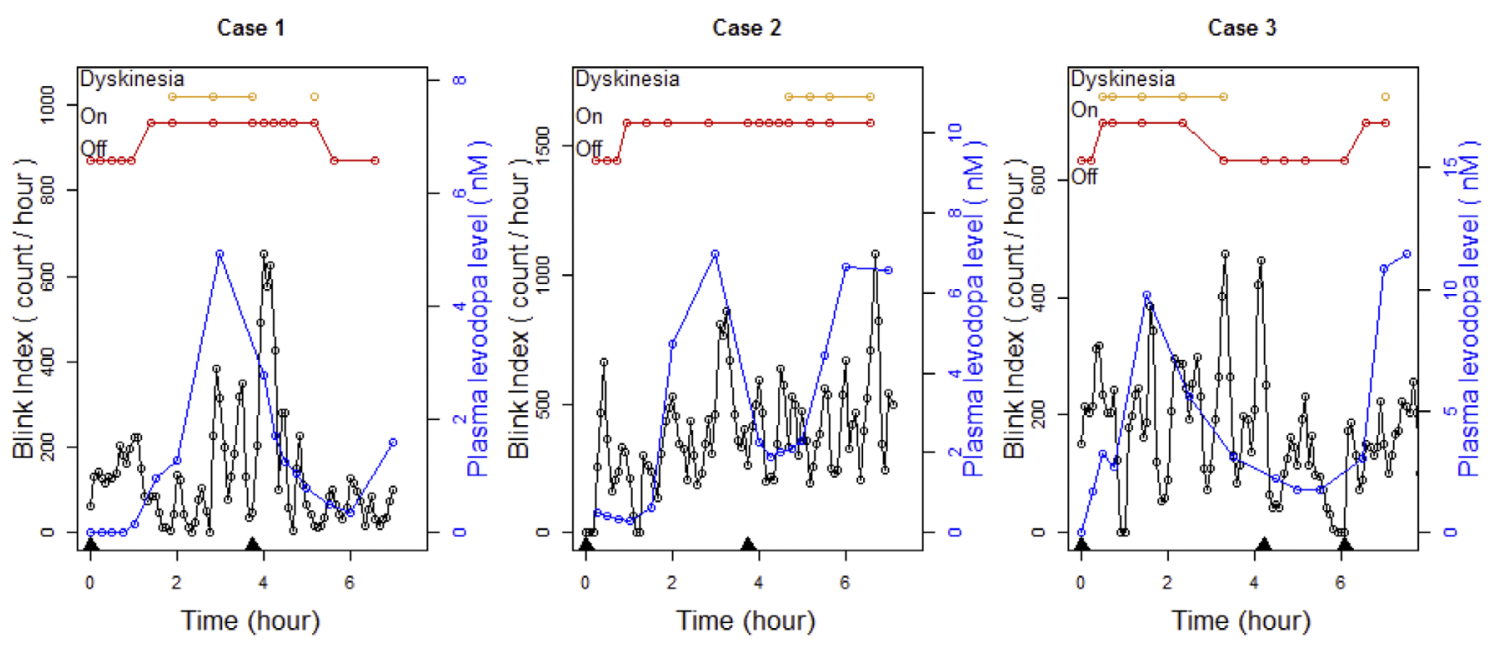

Figure 1. The Blink Index is shown in black dots, plasma levodopa levels are shown in blue dots, motor status of On/Off (wearing-off) is shown in red dots and dyskinesia status is shown in orange dots. The black triangles at the bottom show the oral administration of levodopa.
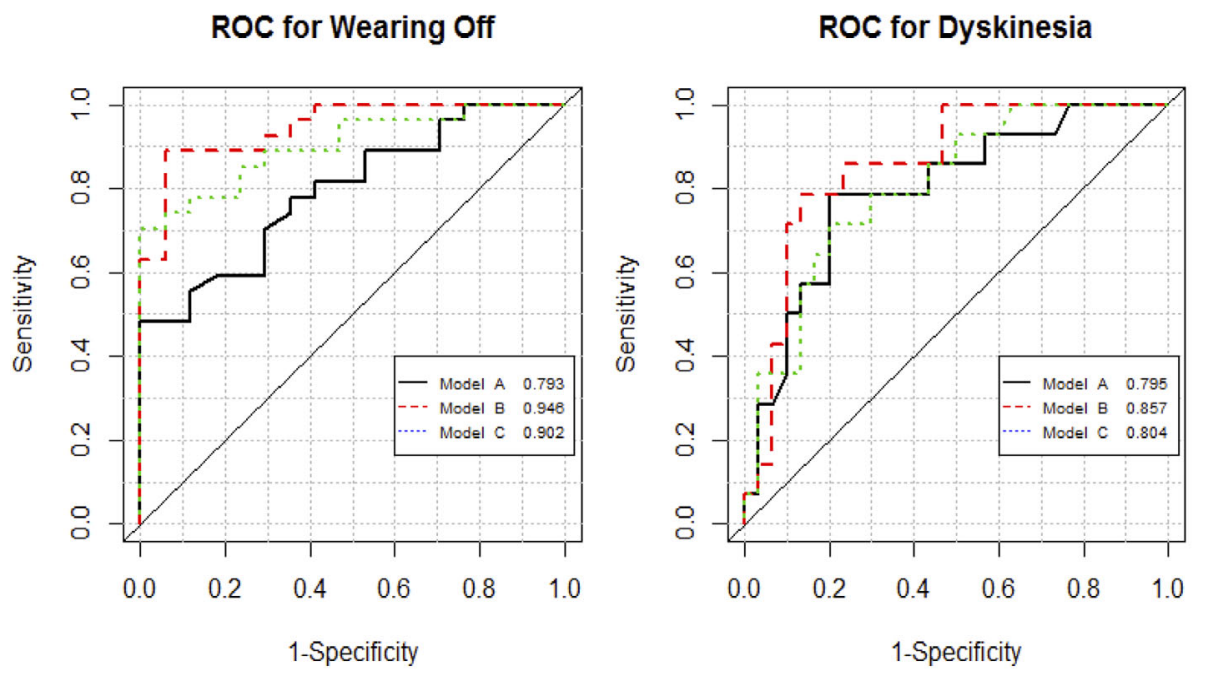

Figure 2. Model A: Modeling using the Blink Index (line), Model B: Modeling using the levodopa levels (dashed line), Model C: Modeling using the Blink Index with adjustment for the first one hour (dotted line).

the first hour of the observation, the AUC improved to 0.902 and did not differ from that of the levodopa level to a statistically significant extent $(\mathrm{p}=0.11)$. For the prediction of dyskinesia, the AUC of the model including the EBR was 0.795 , which was not significantly different from the value of the model based on the levodopa level (ROC of $0.857, \mathrm{p}=$ 0.45). Adjusting for the first hour of the observation period did not improve the prediction of dyskinesia $(\mathrm{p}=0.804)$.

\section{Discussion}

In the present study, we evaluated the clinical significance of spontaneous EBRs for monitoring the motor fluctuations of patients with PD based on EOG data, motor symptoms, and plasma levodopa levels. Although some temporary gaps and fluctuations were observed, our findings suggest that the
Blink Index is correlated with the plasma levodopa level. This finding suggests that EBRs can be used as a substitute for plasma levodopa levels.

Interestingly, when Case 1 experienced the second bout of dyskinesia (time $=5.5 \mathrm{~h}$ ) while the patient's plasma levodopa levels were low, we observed a slight increase in the EBR. This indicates that the EBR may reflect dopaminergic activity in the brain more accurately than the plasma levodopa level. The overall dopaminergic activity in the central nervous system (CNS) is modified not only by levodopa, but also by dopamine agonists, in addition to the secretion of intrinsic dopamine. Also, the levodopa level in the peripheral blood has some lag time because levodopa needs to penetrate the blood-brain barrier and convert to dopamine before action. Thus, only monitoring the plasma levodopa level is insufficient. 
The Blink Index was less successful in detecting wearingoff than plasma levodopa levels. However, this decrease in discriminatory power was mainly due to relatively high EBRs during the first hour of the observation period. During this period, a research nurse physically accompanied the patients-checking the initial settings of the device and drawing blood samples every 15 minute-resulting in continuous interaction with the patient. In addition, none of the included patients wore eyeglasses, indicating that they may have required some time to acclimatize to the device. These factors may have accounted for the differences in the EBR between the first hour and the remainder of the observation period. In future studies, it would probably be useful to give the patients some time to acclimatize to the device before actual observation.

In previous studies regarding spontaneous EBRs in patients with $\mathrm{PD}$, the mean EBR per minute varied, ranging from 5.1-53 (5, 7, 9-11). Although the average EBRs were lower in our patients, we utilized different recording conditions. In all previous studies, recordings were obtained under restricted circumstances (e.g., fixed gaze, during conversation, or watching films), and the duration of observation was as short as a few minutes. Alertness is among the most important determinants of the EBR (12). Thus, a longer observation may have resulted in lower EBRs due to decreases in attention levels. In addition, some previous studies have described a subgroup of patients exhibiting "higher than normal" EBRs (13-15). Levodopa has been reported to reduce EBRs in such patients (15). As our small sample did not include such patients, further research is required. Our data also suggest the need for caution when defining "higher than normal" EBRs, as EBRs appear to vary considerably even within the same individual and across short observation periods. Thus, selecting a single point as the baseline may result in regression to the mean.

Our study did not include patients exhibiting an apparent discrepancy between their plasma levodopa levels and motor status, who are sometimes encountered in clinical practice. Further studies are required to examine how EBRs may be altered in these patients. In addition, although our study conditions were much less restrictive than those utilized in previous studies, larger studies involving a broader range of activities and levels of alertness should be conducted to assess the validity and usefulness of EBRs for monitoring PD. Our study is also limited in that we did not directly count blinks. As EOGs are susceptible to noise, we may have missed or over-counted blinks. However, as long as these errors occurred at random, our estimates remain valid. The similarity of our findings for EBRs and levodopa levels support this assumption.

Recently various portable/wearable devices have been studied to assess the motor status of PD patients (16-19). Most have used accelerometers or gyroscopes to detect movement. These kinematic sensors are not always sensitive or specific enough in the wide range of daily activities. The current gold standard for the assessment of daily motor status is still a patient's diary. Our study is valuable in that we provided the feasibility of using the EBR as another source of diagnostic information complementary to kinematic sensors.

In conclusion, the present study demonstrated that an EBR analysis represents a non-invasive, practical surrogate for measuring the plasma levels of levodopa in patients with PD. In addition, our results suggest that the EBR can be used as a biomarker of motor fluctuation and dyskinesia.

\section{Author's disclosure of potential Conflicts of Interest (COI).}

Haruhiko Morita: Leadership position, EdiGENE Corporation. Masahiro Nomoto: Honoraria, Dainippon Sumitomo Pharma, Hisamitsu Pharmaceutical, Kyowa Hakko Kirin, Novartis Pharma and Otsuka Pharmaceutical.

\section{Financial Support}

Hirotaka Iwaki received a scholarship from The Japanese Society of Clinical Pharmacology and Therapeutics.

\section{References}

1. Contin M, Martinelli P. Pharmacokinetics of levodopa. J Neurol 257: S253-S261, 2010.

2. Astarloa R, Mena MA, Sánchez V, de la Vega L, de Yébenes JG. Clinical and pharmacokinetic effects of a diet rich in insoluble fiber on parkinson disease. Clin Neuropharmacol 15: 375-380, 1992.

3. Doi H, Sakakibara R, Sato M, et al. Plasma levodopa peak delay and impaired gastric emptying in Parkinson's disease. J Neurol Sci 319: 86-88, 2012.

4. Iwaki $\mathrm{H}$, Nishikawa $\mathrm{N}$, Nagai $\mathrm{M}$, et al. Pharmacokinetics of levodopa/benserazide versus levodopa/carbidopa in healthy subjects and patients with Parkinson's disease. Neurol Clin Neurosci 3: 6873, 2014.

5. Karson CN, Burns RS, LeWitt PA, Foster NL, Newman RP. Blink rates and disorders of movement. Neurology 34: 677-678, 1984.

6. Karson CN. Spontaneous eye-blink rates and dopaminergic systems. Brain 106 (Pt 3): 643-653, 1983.

7. Bologna M, Fasano A, Modugno N, Fabbrini G, Berardelli A. Effects of subthalamic nucleus deep brain stimulation and L-DOPA on blinking in Parkinson's disease. Exp Neurol 235: 265-272, 2012.

8. Tomlinson CL, Stowe R, Patel S, Rick C, Gray R, Clarke CE. Systematic review of levodopa dose equivalency reporting in Parkinson's disease. Mov Disord 25: 2649-2653, 2010.

9. Deuschl G, Goddemeier C. Spontaneous and reflex activity of facial muscles in dystonia, Parkinson's disease, and in normal subjects. J Neurol Neurosurg Psychiatry 64: 320-324, 1998.

10. Kimber TE, Thompson PD. Increased blink rate in advanced Parkinson's disease: a form of "off"-period dystonia? Mov Disord 15: 982-985, 2000.

11. Korošec M, Zidar I, Reits D, Evinger C, VanderWerf F. Eyelid movements during blinking in patients with Parkinson's disease. Mov Disord 21: 1248-1251, 2006.

12. Jongkees BJ, Colzato LS. Spontaneous eye blink rate as predictor of dopamine-related cognitive function-a review. Neurosci Biobehav Rev 71: 58-82, 2016.

13. Karson CN, LeWitt PA, Calne DB, Wyatt RJ. Blink rates in parkinsonism. Ann Neurol 12: 580-583, 1982.

14. Kimber TE, Thompson PD. Increased blink rate in advanced Parkinson's disease: a form of 'off'-period dystonia? Mov Disord 
15: 982-985, 2000.

15. Agostino R, Bologna M, Dinapoli L, et al. Voluntary, spontaneous, and reflex blinking in Parkinson's disease. Mov Disord 23: 669675, 2008.

16. Mitoma H, Yoneyama M, Orimo S. 24-hour recording of parkinsonian gait using a portable gait rhythmogram. Intern Med 49: 2401-2408, 2010.

17. Iijima M, Mitoma H, Uchiyama S, Kitagawa K. Long-term monitoring gait analysis using a wearable device in daily lives of patients with Parkinson's disease: the efficacy of selegiline hydrochloride for gait disturbance. Front Neurol 8: 542, 2017.

18. Rovini E, Maremmani C, Cavallo F. How wearable sensors can support Parkinson's disease diagnosis and treatment: a systematic review. Front Neurosci 11: 555, 2017.

19. Rodríguez-Molinero $\mathrm{A}$, Pérez-López $\mathrm{C}$, Samà $\mathrm{A}$, et al. $\mathrm{A}$ kinematic sensor and algorithm to detect motor fluctuations in Parkinson disease: validation study under real conditions of use. JMIR Rehabil Assist Technol 5: e8, 2018.

The Internal Medicine is an Open Access journal distributed under the Creative Commons Attribution-NonCommercial-NoDerivatives 4.0 International License. To view the details of this license, please visit (https://creativecommons.org/licenses/ by-nc-nd/4.0/).

(C) 2019 The Japanese Society of Internal Medicine Intern Med 58: 1417-1421, 2019 